From the Graduate Institute of Epidemiology, College of Public Health, National Taiwan University; Department of Gastroenterology, Ren-Ai Branch, Taipei City Hospital; Division of Gastroenterology, Department of Internal Medicine, National Taiwan University Hospital and National Taiwan University College of Medicine; and the Department of Laboratory Medicine, National Taiwan University Hospital and Genomics Research Center, Academia Sinica, Taipei, Taiwan

Submitted January 5, 2008; accepted July 17, 2008; published online ahead of print at www.jco.org on October 27 2008

Supported by Grants No. NSC94-3112 B-002-017, NSC95-3112-B-002-001, and NSC95-2314-B-002-244-MY3 from the National Science Council, Taiwan.

Authors' disclosures of potential conflicts of interest and author contributions are found at the end of this article.

Corresponding author: Ming-Whei Yu, $\mathrm{PhD}$, Graduate Institute of Epidemiology, College of Public Health, National Taiwan University, Room 522, No.17, Xuzhou Rd, Zhongzheng District, Taipei City 10055, Taiwan; e-mail: yumw@ntu.edu.tw.

(C) 2008 by American Society of Clinical Oncology

0732-183X/08/2634-5576/\$20.00

DOI: 10.1200/JCO.2008.16.1075

\title{
Body-Mass Index and Progression of Hepatitis B: A Population-Based Cohort Study in Men
}

Ming-Whei Yu, Wei-Liang Shih, Chih-Lin Lin, Chun-Jen Liu, Jhih-Wei Jian, Keh-Sung Tsai, and Chien-Jen Chen

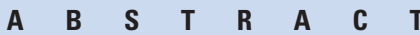

\section{Purpose}

To determine prospectively whether body-mass index (BMI) is associated with liver-related morbidity and mortality among male hepatitis B virus (HBV) carriers

\section{Patients and Methods}

We performed a prospective study of 2,903 male HBV surface antigen-positive government employees who were free of cancer at enrollment between 1989 and 1992. Main outcome measures included ultrasonography, biochemical tests, incident hepatocellular carcinoma (HCC), and liver-related death.

\section{Results}

During mean follow-up of 14.7 years, 134 developed HCC and 92 died as a result of liver-related causes. In Cox proportional hazards models adjusting for age, number of visits, diabetes, and use of alcohol and tobacco, the hazard ratios for incident $\mathrm{HCC}$ were $1.48(95 \% \mathrm{Cl}, 1.04$ to 2.12) in overweight men (BMI between 25.0 and $\left.29.9 \mathrm{~kg} / \mathrm{m}^{2}\right)$ and $1.96(95 \% \mathrm{Cl}, 0.72$ to 5.38) in obese men $\left(\mathrm{BMI} \geq 30.0 \mathrm{~kg} / \mathrm{m}^{2}\right)$, compared with normal-weight men (BMI between 18.5 and $24.9 \mathrm{~kg} / \mathrm{m}^{2}$ ). Liver-related mortality had adjusted hazard ratios of $1.74(95 \% \mathrm{Cl}, 1.15$ to 2.65$)$ in overweight men and $1.50(95 \% \mathrm{Cl}, 0.36$ to 6.19$)$ in obese men. Excess BMI was also associated with the occurrence of fatty liver and cirrhosis detected by ultrasonography, as well as elevated ALT and $\gamma$-glutamyltransferase (GGT) activity during follow-up. The association of BMI with GGT was stronger than with ALT, and elevated GGT activity and cirrhosis were the strongest predictors for incident HCC and liver-related death.

\section{Conclusion}

This longitudinal cohort study indicates that excess body weight is involved in the transition from healthy HBV carrier state to HCC and liver-related death among men.

\section{J Clin Oncol 26:5576-5582. (C) 2008 by American Society of Clinical Oncology}

\section{INTRODUCTION}

Substantial epidemiologic evidence indicates that diabetes is associated with an increased risk of hepatocellular carcinoma (HCC). ${ }^{1-4}$ Although HCC or liver cancer has also been linked to excess body weight, which has long been recognized as an important cause of diabetes, ${ }^{5}$ our knowledge of the magnitude of the relation in different populations with diverse etiology of HCC is limited. . $^{6-10}$

It is estimated that approximately $80 \%$ to $85 \%$ of individuals with obesity, defined by a body-mass index (BMI) of $30 \mathrm{~kg} / \mathrm{m}^{2}$ or more, have some form of fatty liver disease. ${ }^{11-14}$ However, fatty liver disease resulting from obesity alone generally has a benign clinical course. ${ }^{15-17}$ The potential effect of obesity in persons at high risk for HCC from some other factor, such as hepatitis virus infection and alcohol abuse, remains largely unknown. So far, four pro- spective studies have investigated the possible association between BMI and the development of HCC or liver cancer. Whereas three studies found excess BMI to be a risk factor, ${ }^{6,7,9}$ one observed an effect in only a subgroup. ${ }^{8}$ On the other hand, a large casecontrol study has failed to detect a significant association. ${ }^{10}$ These studies were all conducted in areas of low prevalence of hepatitis B virus (HBV) infection, and did not examine the presence of specific risk factors in patients with HCC.

To assess the role of excess BMI in different natural history phases of hepatitis $\mathrm{B}$, we have conducted a prospective study of a cohort of male HBV carriers who were enrolled during regular physical examination and had been followed for an average of 14.7 years. We sought to determine whether excess BMI is associated with liver-related morbidity and mortality, including cirrhosis and HCC, and to assess the associations between BMI and serum 
markers of inflammation and oxidative stress, such as aminotransferase and $\gamma$-glutamyltransferase (GGT) activity, ${ }^{18}$ during longterm follow-up.

\section{PATIENTS AND METHODS}

\section{The Cohort}

This study is part of an ongoing prospective study focusing on the etiology of HCC, in which a total of 4,841 HBV surface antigen (HBsAg)positive men were recruited from two sources: Government Employees' Central Clinics and the Liver Unit of Chang Gung Memorial Hospital (Taipei, Taiwan). ${ }^{19,20}$ The population in the present study consisted of $2903 \mathrm{HBsAg-}$ positive male government employees who were enrolled during routine free physical examination between August 1989 and June 1992. This subcohort was chosen for the present study because cardiovascular disease risk factors were also examined (Fig 1). Its follow-up was achieved through two methods: (1) directly, by means of medical examinations including ultrasonography and blood testing; (2) indirectly, through secondary sources of information including medical records and national cancer and death registries. Study participants were invited to return for follow-up examinations every year and more frequently if indicated. This study was approved by the research ethics committee at the College of Public Health, National Taiwan University (Taipei, Taiwan). All participants provided informed consent.

\section{Data Collection}

At recruitment, the workup consisted of a physical examination by a clinic physician, blood draw, blood pressure measurement, and anthropometry. Height and weight were measured on a standard physician's balance beam scale and stadiometer. BMI was calculated using weight divided by height squared $\left(\mathrm{kg} / \mathrm{m}^{2}\right)$. All study participants were tested for HBsAg and serum ALT and alpha-fetoprotein. Antibodies against hepatitis $\mathrm{C}$ virus (anti-HCV) were assayed when blood samples were available. Serum HBsAg was tested by a radioimmunoassay (Abbott Laboratories, North Chicago, IL), and anti-HCV was assayed by a second-generation enzyme immunoassay (Abbott Laboratories). Serum alpha-fetoprotein was measured by an enzyme-linked immunosorbent assay (Abbott Laboratories). Information on sociodemographic

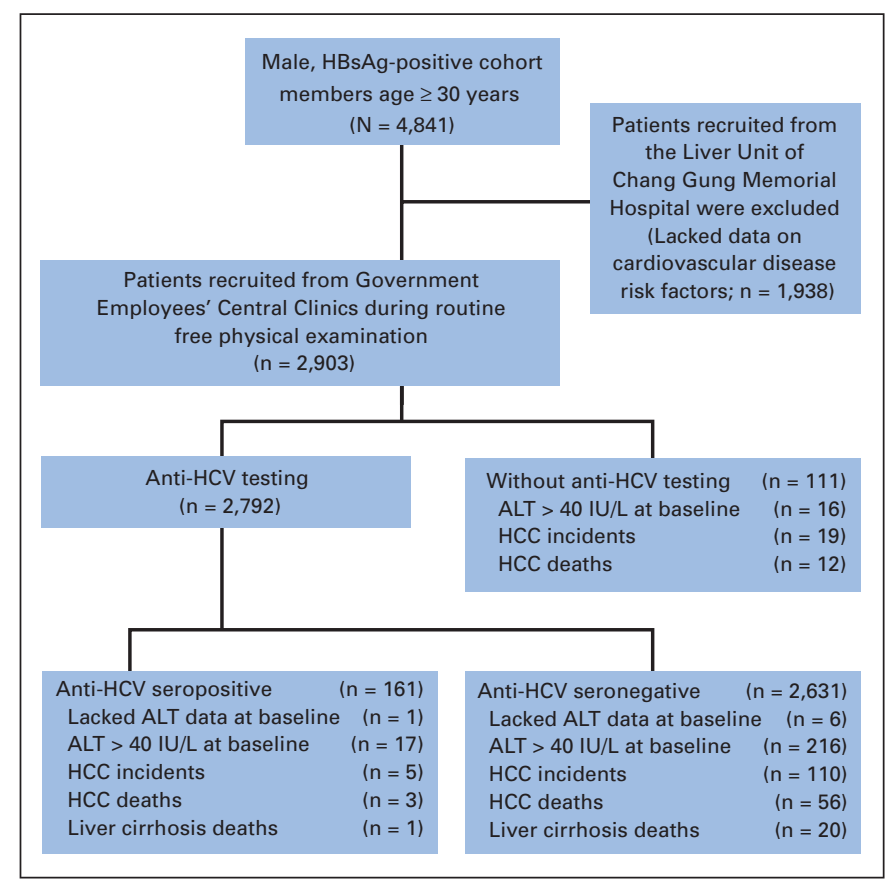

Fig 1. Enrollment and outcomes. HCC, hepatocellular carcinoma; HCV, hepatitis $C$ virus; HBsAg, hepatitis $B$ virus surface antigen. characteristics, lifestyle habits, and medical history were obtained by in-person interviews according to a structured questionnaire.

During follow-up, high-resolution, real-time ultrasonography was performed routinely since 1993 for the detection of liver abnormalities, such as fatty liver, cirrhosis, and HCC. Blood tests during follow-up included alphafetoprotein, ALT, AST, GGT, albumin, globulin, cholesterol, triglycerides, creatinine, uric acid, fasting glucose, and high-density lipoprotein cholesterol (HDL-C). Serum ALT and AST were examined routinely after August 1994; GGT, cholesterol, and triglycerides after August 1996; and fasting glucose and HDL-C after February 2005. The triglycerides:HDL-C ratio of 3.5 or greater was used to identify individuals with insulin resistance. ${ }^{21}$

\section{Statistical Analysis}

BMI was divided into four categories according to the WHO cut points with corresponding interpretations ( $<18.5 \mathrm{~kg} / \mathrm{m}^{2}$, underweight; 18.5 to 24.9 $\mathrm{kg} / \mathrm{m}^{2}$, normal weight; 25.0 to $29.9 \mathrm{~kg} / \mathrm{m}^{2}$, overweight; $\geq 30.0 \mathrm{~kg} / \mathrm{m}^{2}$, obese) $)^{5}$ or according to the quartile levels of our study participants. We used the $\chi^{2}$ and Fisher's exact tests to compare groups for categoric variables, as appropriate. Both time-fixed and -dependent Cox models were used to evaluate the association of potential risk factors with incident HCC and liver-related deaths. In the time-fixed model, only initial measurements (eg, BMI) were applied. The time-dependent model used the repeated measurements of variables during follow-up (eg, liver biochemical tests and ultrasonography measurements). Odds ratios (ORs) and 95\% CIs from unconditional logistic regression models were used to assess the associations between BMI and abnormalities diagnosed by ultrasonography or biochemical tests. Multivariate models were adjusted for age at recruitment, number of visits, diabetes, and habits of tobacco and alcohol use, unless otherwise stated. Habitual alcohol consumption was defined as drinking alcohol at least 1 day per week for at least 1 year. To examine the possible impact of missing data for fasting glucose and the ratio of triglycerides to HDL-C, we compared proportions of missing data on the two variables by BMI categories. Under the assumption of missing at random, multiple imputation was applied to assess the robustness of the results to missing data. We used a logistic regression model to predict values of the two variables. ${ }^{22}$ The imputer's model was performed using the same set of covariates as the model of complete case analysis. All analyses were performed with SAS software, version 9.1 (SAS Institute, Cary, NC).

\section{RESULTS}

Table 1 lists the baseline characteristics in the 2,903 male HBsAg carriers by BMI. There was a significant positive linear trend in the proportion of elevated serum ALT activity with increasing BMI (Mantel's $\chi^{2}$ test $P=.0001$ ). The distributions of alcohol consumption $(P=.0060)$ and cigarette smoking $(P=.0079)$ varied to a statistically significant extend according to BMI. However, the proportion of alcohol consumed greater than level of $140 \mathrm{~g} / \mathrm{wk}$ was no more than $10 \%$ in any BMI group. Overall, $2.5 \%$ of men had a history of diabetes. A higher proportion of obese men than leaner men had a history of diabetes $(P=.0033)$. Differences between BMI groups for age, first-degree family history of HCC, and anti-HCV positivity generally were small.

The vital status and cancer occurrence of cohort members were determined from 1989 to 1992 through December 31, 2005. During average follow-up of 14.7 ( \pm 2.5 ) years, a total of 134 HCC cases (five were anti-HCV positive) diagnosed by histologic findings and/or an elevated level of serum alpha-fetoprotein ( $\geq 400 \mathrm{ng} / \mathrm{mL}$ ) combined with at least one positive image from angiography, sonography, and/or computed tomography were observed. There were 218 deaths, including 92 (42.2\% of total deaths) attributable to liver-related disease. HCC was the cause of death in 71 men (three were anti-HCV positive) and liver cirrhosis in 21 (one was anti-HCV positive; Fig 1). 


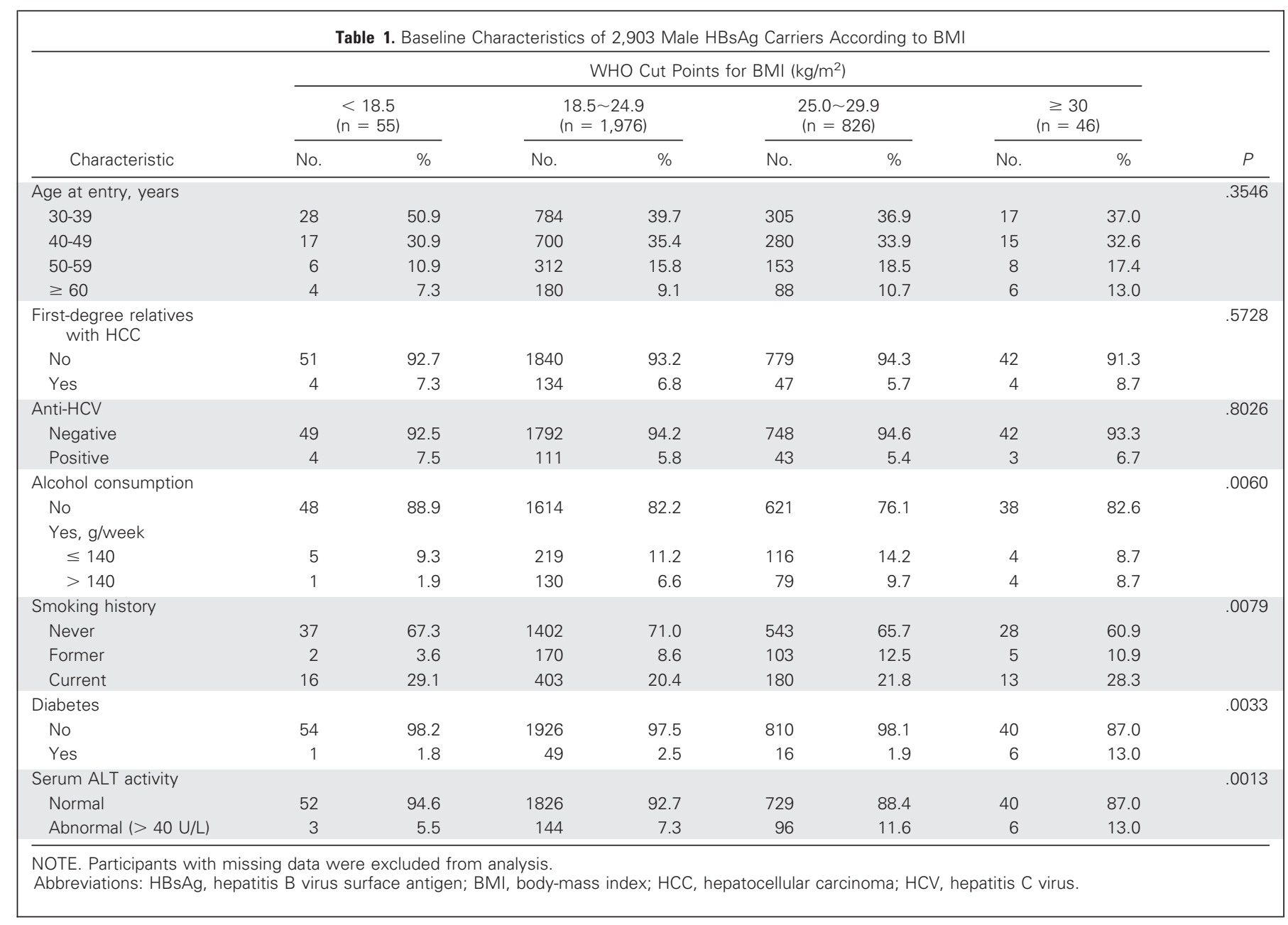

The number of visits was not significantly different between BMI groups. In Cox proportional hazards models adjusting for age, number of visits, diabetes, and use of alcohol and tobacco, a tendency toward greater risk for incident $\operatorname{HCC}\left(P_{\text {trend }}=.0479\right)$ and liver-related deaths $\left(P_{\text {trend }}=.0011\right)$ was observed in higher quartile of BMI. Analyses on the WHO cut points for BMI showed that the hazard ratios (HRs) for incident HCC were 1.48 (95\% CI, 1.04 to 2.12) in overweight men and 1.96 (95\% CI, 0.72 to 5.38 ) in obese men compared with normal-weight men. No underweight men died as a result of liver disease. Liver-related mortality had HRs of 1.74 (95\% CI, 1.15 to 2.65) in overweight men and 1.50 (95\% CI, 0.36 to 6.19) for obese men (Table 2). Similar results were seen when we excluded participants who were positive or missing for anti-HCV (data not shown).

In contrast, there was no significant effect of diabetes on the development of HCC (HRs were 1.14 [95\% CI, 0.55 to 2.39] after adjusting quartiles of BMI and other potential confounders and 1.16 [95\% CI, 0.55 to 2.42] when using WHO cut points for BMI) or liver-related death (HRs were 1.35 [95\% CI, 0.61 to 3.00] after adjusting quartiles of BMI and other potential confounders and 1.37 [95\% CI, 0.62 to 3.05] when using WHO cut points for BMI).

Male HBsAg carriers with excess BMI at entry were more likely than normal weight or underweight to have elevated serum ALT and
GGT activity, as well as reduced AST:ALT ratio during follow-up. After adjustment for other potential confounders, these associations remained statistically significant (Table 3).

Fatty liver was detected by ultrasonography in $65.0 \%$ of normalweight male HBsAg carriers compared with $83.6 \%$ overweight and 93.2\% obese carriers. Only $28.9 \%$ of male HBsAg carriers who were underweight had fatty liver. A total of 257 male HBsAg carriers with cirrhosis detected by ultrasonography were identified during follow-up. The risks of both fatty liver $\left(P_{\text {trend }}<.0001\right)$ and cirrhosis $\left(P_{\text {trend }}=.0005\right)$ increased with increasing quartiles of BMI. Using WHO cut points for BMI, the adjusted ORs increased to 9.72 (95\% CI, 2.96 to 31.97 ) for fatty liver and 2.37 (95\% CI, 1.05 to 5.35 ) for cirrhosis among male HBsAg carriers of obesity, compared with those who had normal weight (Table 3).

With the exception of hypercholesterolemia (cholesterol $>200$ $\mathrm{mg} / \mathrm{dL}$ ), every other risk factor for cardiovascular disease (including triglycerides $>200 \mathrm{mg} / \mathrm{dL}$, fasting glucose $\geq 110 \mathrm{mg} / \mathrm{dL}$, and insulin resistance determined by the triglycerides:HDL-C ratio $\geq 3.5$ ) was associated with excess BMI. After adjusting potential confounders such as age, use of alcohol and tobacco, diabetes (for hypertriglyceridemia only), and number of visits, hypertriglyceridemia and hyperglycemia were associated with elevated ALT (ORs were 1.26 [95\% CI, 


\begin{tabular}{|c|c|c|c|c|c|c|c|c|c|}
\hline \multirow[b]{2}{*}{ Characteristic } & \multicolumn{4}{|c|}{ WHO Cut Points for BMI $\left(\mathrm{kg} / \mathrm{m}^{2}\right)$} & \multicolumn{4}{|c|}{ Quartile of BMI $\left(\mathrm{kg} / \mathrm{m}^{2}\right)$} & \multirow[b]{2}{*}{$\begin{array}{l}P \text { for } \\
\text { Trend" }\end{array}$} \\
\hline & $\begin{array}{c}<18.5 \\
(n=55)\end{array}$ & $\begin{array}{l}18.5 \sim 24.9 \\
(n=1976)\end{array}$ & $\begin{array}{l}25.0 \sim 29.9 \\
(n=826)\end{array}$ & $\begin{array}{c}\geq 30 \\
(n=46)\end{array}$ & $\begin{array}{c}<22.0 \\
(n=727)\end{array}$ & $\begin{array}{l}22.0 \sim 23.6 \\
(n=716)\end{array}$ & $\begin{array}{l}23.7 \sim 25.3 \\
(n=730)\end{array}$ & $\begin{array}{c}\geq 25.4 \\
(n=730)\end{array}$ & \\
\hline \multicolumn{10}{|l|}{ No. of visits } \\
\hline Median & 7 & 8 & 8 & 6.5 & 8 & 8 & 8 & 8 & \\
\hline $\begin{array}{l}\text { Interquartile } \\
\text { range }\end{array}$ & 4 to 9 & 5 to 9 & 5 to 9 & 4 to 8 & 5 to 9 & 5 to 9 & 5 to 9 & 5 to 9 & \\
\hline Mean & $6.5( \pm)$ & 7.0 & 7.0 & $6.3( \pm)$ & 7.0 & 7.0 & 7.0 & 6.9 & \\
\hline $\begin{array}{l}\text { Standard } \\
\text { deviation }\end{array}$ & 3.3 & 2.8 & 2.8 & 2.5 & 2.8 & 2.7 & 2.8 & 2.8 & \\
\hline$P+$ & \multicolumn{4}{|c|}{.2100} & \multicolumn{4}{|c|}{.8346} & \\
\hline \multicolumn{10}{|l|}{$\mathrm{HCC}$} \\
\hline No. of cases & 3 & 77 & 50 & 4 & 28 & 31 & 29 & 46 & \\
\hline Incidence rate $\neq$ & 367.3 & 263.4 & 416.2 & 616.6 & 258.5 & 294.4 & 268.6 & 435.9 & \\
\hline Hazard ratio§ & 1.55 & 1.0 & 1.48 & 1.96 & 1.0 & 1.06 & 1.06 & 1.58 & .0479 \\
\hline $95 \% \mathrm{Cl}$ & 0.49 to 4.93 & & 1.04 to 2.12 & 0.72 to 5.38 & & 0.63 to 1.76 & 0.63 to 1.79 & 0.99 to 2.53 & \\
\hline \multicolumn{10}{|l|}{ Liver-related deaths } \\
\hline No. of deaths & 0 & 51 & 39 & 2 & 13 & 21 & 22 & 36 & \\
\hline Death rateł & 0 & 172.6 & 319.8 & 302.8 & 118.4 & 197.3 & 201.7 & 335.7 & \\
\hline Hazard ratio§ & NA & 1.0 & 1.74 & 1.50 & 1.0 & 1.52 & 1.79 & 2.69 & .0011 \\
\hline $95 \% \mathrm{Cl}$ & NA & & 1.15 to 2.65 & 0.36 to 6.19 & & 0.76 to 3.03 & 0.90 to 3.55 & 1.43 to 5.07 & \\
\hline \multicolumn{10}{|l|}{$\begin{array}{l}\text { All other causes of } \\
\text { death }\end{array}$} \\
\hline No. of deaths & 2 & 78 & 42 & 4 & 22 & 33 & 29 & 42 & \\
\hline Death rate & 236.7 & 263.9 & 344.4 & 605.5 & 200.4 & 310.1 & 265.8 & 391.7 & \\
\hline Hazard ratio§ & 1.24 & 1.0 & 1.22 & 1.75 & 1.0 & 1.44 & 1.50 & 1.93 & .0141 \\
\hline $95 \% \mathrm{Cl}$ & 0.30 to 5.08 & & 0.84 to 1.79 & 0.64 to 4.82 & & 0.83 to 2.49 & 0.85 to 2.63 & 1.14 to 3.27 & \\
\hline \multicolumn{10}{|c|}{$\begin{array}{l}\text { Abbreviations: HCC, hepatocellular carcinoma; BMI, body-mass index; HBsAg, hepatitis B virus surface antigen; NA, not applicable. } \\
\text { *Trend tests for quartiles of BMI in the Cox models were performed by assigning the medians of each category as the score. } \\
+P \text { values are for the difference in the means between BMI groups. } \\
\text { †The rate per } 100,000 \text { person-year is given. } \\
\text { §Hazard ratios were adjusted for age at recruitment, number of visits, cigarette smoking, alcohol consumption, and history of diabet }\end{array}$} \\
\hline
\end{tabular}

1.00 to 1.58 ] for hypertriglyceridemia and 1.73 [95\% CI, 1.29 to 2.33 ] for hyperglycemia) and GGT (ORs were 2.00 [95\% CI, 1.54 to 2.59] for hypertriglyceridemia and 2.12 [95\% CI, 1.50 to 2.99] for hyperglycemia) during follow-up. The index of insulin resistance was associated with elevated GGT (OR $=1.64 ; 95 \% \mathrm{CI}, 1.20$ to 2.24 ) but not ALT. All cardiovascular disease risk factors were positively associated with fatty liver. However, although hypercholesterolemia had an inverse association with cirrhosis, no other risk factors of cardiovascular disease were statistically significantly associated with cirrhosis. The proportion of missing data on fasting glucose and the triglycerides: HDL-C ratio were $43.6 \%, 43.5 \%, 46.6 \%$, and $54.4 \%$, respectively, for underweight, normal-weight, overweight, and obese male HBsAg carriers $(P=.2451)$. The imputation analysis yielded similar results as using complete case analysis (data not shown).

By multivariate Cox regressions, cirrhosis diagnosed by ultrasonography, ALT, AST, and GGT were significantly, positively associated with increased risk for incident HCC and liver-related death, whereas fatty liver was associated with a lower risk for both outcomes (Table 4). When ALT and GGT, the two liver enzymes associated with BMI, were included in the same models, GGT was a stronger predictor for both incident HCC (HRs were 2.68 [95\% CI, 1.50 to 4.80] for elevated ALT and 3.45 [95\% CI, 1.88 to 6.36] for elevated GGT) and liver-related death (HRs were 1.64 [95\% CI, 0.78 to 3.47] for elevated ALT and 14.06 [95\% CI, 6.45 to 30.63] for elevated GGT) than ALT.

\section{DISCUSSION}

Using different cut points for BMI, this cohort study performed in HBsAg carriers shows that excess weight increased risk for developing advanced liver disease, including HCC and cirrhosis. Liver-related disease accounted for $42 \%$ of the deaths in HBV carriers, and overweight men had a risk of death from liver-related disease almost two times as great as men of normal weight. We also observed that high BMI was associated with elevated serum ALT and GGT activity, which are significant predictors of risk for incident HCC and liver-related death.

We conducted this study among male government employees because their prevalence of excess consumption of alcohol, an important cause of cirrhosis and HCC, ${ }^{23,24}$ is low. Indeed, only a small minority of the study participants consumed alcohol at a level greater than $140 \mathrm{~g} / \mathrm{wk}$, which is much less than the minimum alcohol intake required for an hepatotoxic effect in men. ${ }^{23}$ The adjustment for habitual alcohol drinking by use of multivariate analysis further make us confident in excluding bias induced by confounding effect of alcohol drinking on the association between BMI and hepatic disease.

Obesity is causally linked to the development of type 2 diabetes mellitus, ${ }^{5}$ which has been associated with increased risk of both chronic liver disease and liver cancer or HCC. ${ }^{1-4}$ The biologic mechanisms by which diabetes may lead to significant liver disease are still unclear. However, some experimental studies have found persuasive 


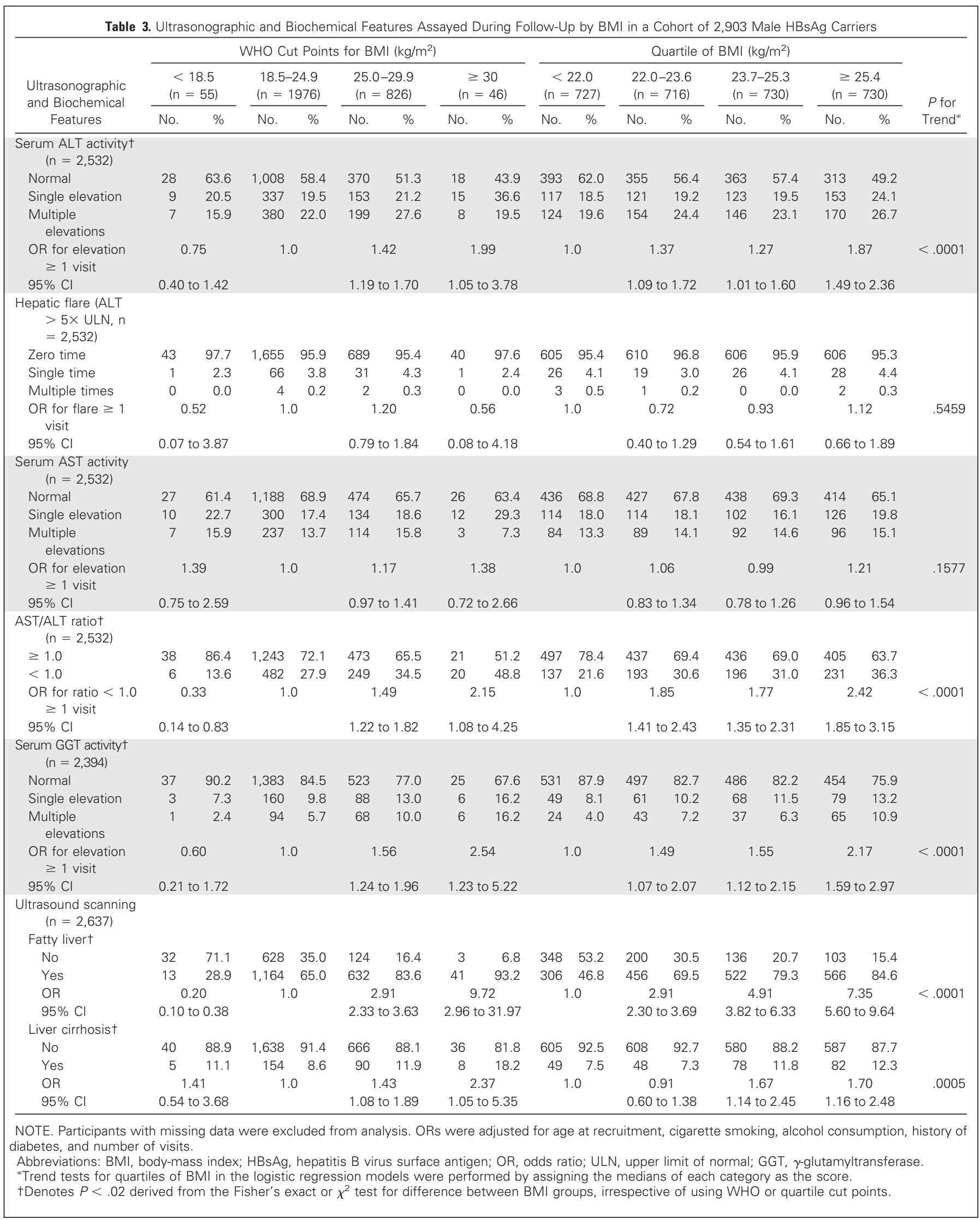


Table 4. Associations Between Clinical Indices of Hepatic Abnormalities and Risk of Incident HCC and Liver-Related Death in a Cohort of 2,903 Male HBsAg Carriers

\begin{tabular}{|c|c|c|c|c|}
\hline Index & Hazard Ratio & $\mathrm{HCC} 95 \% \mathrm{Cl}$ & Hazard Ratio & $\begin{array}{l}\text { Liver-Related Death } \\
\qquad 95 \% \mathrm{Cl}\end{array}$ \\
\hline Elevated ALT at baseline* & 4.14 & 2.78 to 6.18 & 4.77 & 3.01 to 7.57 \\
\hline \multicolumn{5}{|l|}{ During follow-up } \\
\hline Elevated ALT† & 3.89 & 2.44 to 6.19 & 4.52 & 2.46 to 8.32 \\
\hline Hepatic flare $(A L T>5 \times$ ULN) $\neq$ & 4.56 & 1.94 to 10.70 & 7.27 & 2.96 to 17.82 \\
\hline Elevated AST† & 3.90 & 2.44 to 6.23 & 7.64 & 4.24 to 13.79 \\
\hline Elevated GGT† & 5.23 & 2.99 to 9.14 & 17.57 & 8.74 to 35.32 \\
\hline \multicolumn{5}{|l|}{ Ultrasound scanning $†$} \\
\hline Fatty liver & 0.24 & 0.14 to 0.42 & 0.18 & 0.08 to 0.41 \\
\hline Liver cirrhosis & 14.81 & 9.88 to 22.20 & 27.18 & 16.15 to 45.73 \\
\hline
\end{tabular}

Abbreviations: HCC, hepatocellular carcinoma; HBsAg, hepatitis B virus surface antigen; ULN, upper limit of normal; GGT, $\gamma$-glutamyltransferase.

*Hazard ratios were calculated by a time-fixed Cox model adjusting for age at recruitment, cigarette smoking, and alcohol consumption.

tHazard ratios were calculated by modeling time-varying effects of repeated measurements of abnormal test results in the Cox models with adjustment for age at recruitment, cigarette smoking, alcohol consumption, and number of visits.

¥Hazard ratio was calculated by a Cox model using the first occurrence of hepatic flare as a time-dependent variable with adjustment for age at recruitment, cigarette smoking, alcohol consumption, and number of visits.

evidence that high glucose levels or insulin resistance, which play a primary role in the development of type 2 diabetes mellitus, ${ }^{25,26}$ may promote hepatic fibrogenesis. ${ }^{27-29}$ Further, insulin resistance has been associated with elevated ALT activity in persons with different etiology of chronic liver disease, ${ }^{30}$ and with fibrosis in persons with hepatitis $\mathrm{C}$ or nonalcoholic fatty liver disease. ${ }^{31}$

We found that elevated fasting glucose levels but not the index of insulin resistance determined by the triglycerides:HDL-C ratio of 3.5 or greater were associated with increased ALT activity. However, elevated fasting glucose levels and the index of insulin resistance had similar association with elevated GGT activity. In addition, BMI and fasting glucose levels were more strongly associated with GGT than with ALT. Elevated GGT is a marker of central fat accumulation, and has been linked to moderate/severe steatosis in recent studies. ${ }^{32,33}$ Therefore, the increased GGT may reflect an association between excess weight and the severity of steatosis.

In contrast to the aforementioned findings from experimental models on the mechanisms of fibrosis, ${ }^{27-29}$ we failed to find an association between sonographic evidence of cirrhosis, the end-stage consequence of fibrosis, and fasting glucose levels or insulin resistance. Also, we did not find an association between history of diabetes and incident HCC or liver-related death. Only $2.5 \%$ of our entire sample reported a history of diabetes at enrollment. In multivariate analyses with BMI, history of diabetes, and all other conventional risk factors as covariates, higher BMI was associated with statistically significant increase in the risks of both incident HCC and liver-related death, whereas no such associations were found for diabetes. Furthermore, the associations between BMI and cirrhosis or elevated ALT or GGT activity remained statistically significant even after adjusting for diabetes. We thus hypothesized that excess BMI may predispose HBV carriers to the development of significant liver disease before overt diabetes occurs.

There are various plausible mechanisms for the possible causal relationship between excess weight and HCC, and lipid peroxidation and increased oxidative stress may play a central role. ${ }^{34-36}$ Previous imaging and autopsy studies have indicated that hepatic steatosis is common in obese individuals. ${ }^{11-14}$ In the light of a recent animal model, ${ }^{37}$ the presence of chronic HBV infection may provide a synergistic effect with obesity on the hepatic lipid accumulation and the development of steatosis. Indeed, we found a strong association between higher BMI and fatty liver in HBV carriers. However, fatty liver was not a predictor for the risk of incident HCC or liver-related death. Consistent with this finding, studies conducted in areas with low HCC incidence have shown a benign long-term prognosis in patients who had a histologic diagnosis of pure fatty liver without inflammation. ${ }^{16}$

The development of fatty liver is the earliest stage of nonalcoholic fatty liver disease. The second stage of nonalcoholic fatty liver disease involves oxidative stress and activating an inflammatory response that causes steatohepatitis. ${ }^{34,38}$ Both ALT and GGT have been proposed as markers of inflammation and oxidative stress, ${ }^{18}$ but only GGT is related to the severity of steatosis. ${ }^{33}$ Besides ALT, which has been widely accepted as a routine test for monitoring hepatitis $\mathrm{B}$, we observed that GGT was associated with incident HCC and liver-related death. Moreover, elevated GGT was a stronger predictor for both outcomes than ALT. This finding may imply the importance of the setting of steatohepatitis in hepatitis B progression.

A limitation of this study may be the lack of data on fasting glucose levels and the index of insulin resistance in a high frequency of study participants because serum glucose and HDL-C began to be included as a routine test until recently. However, because missing observations did not appear related to BMI, we expect that missing data decreased the power of our study but did not bias the results. Indeed, we found similar results from analyses restricted to participants with complete data and those from analyses with imputing missing measurement data.

In summary, over a period of 16 years, this longitudinal cohort study suggests that excess weight is involved in the transition from healthy HBV carrier state to HCC and liver-related death among men. The association of higher BMI with increased risk of HCC or death resulting from liver disease is independent of diabetes. The spectrum of liver diseases in relation to excess weight among HBsAg carriers extends from simple fatty liver at the most benign end to chronic hepatitis, cirrhosis, and HCC at the opposite end.

\section{AUTHORS' DISCLOSURES OF POTENTIAL CONFLICTS} OF INTEREST

The author(s) indicated no potential conflicts of interest. 


\section{AUTHOR CONTRIBUTIONS}

Conception and design: Ming-Whei Yu, Wei-Liang Shih, Chih-Lin Lin, Chun-Jen Liu, Jhih-Wei Jian, Keh-Sung Tsai, Chien-Jen Chen Financial support: Ming-Whei Yu
Collection and assembly of data: Ming-Whei Yu, Wei-Liang Shih, Chih-Lin Lin, Chun-Jen Liu, Keh-Sung Tsai, Chien-Jen Chen Data analysis and interpretation: Ming-Whei Yu, Jhih-Wei Jian Manuscript writing: Ming-Whei Yu Final approval of manuscript: Ming-Whei Yu, Wei-Liang Shih, Chih-Lin Lin, Chun-Jen Liu, Jhih-Wei Jian, Keh-Sung Tsai, Chien-Jen Chen

\section{REFERENCES}

1. El-Serag HB, Tran T, Everhart JE: Diabetes increases the risk of chronic liver disease and hepatocellular carcinoma. Gastroenterology 126:460468, 2004

2. Adami HO, Chow WH, Nyren O, et al: Excess risk of primary liver cancer in patients with diabetes mellitus. J Natl Cancer Inst 88:1472-1477, 1996

3. Wideroff L, Gridley G, Mellemkjaer L, et al: Cancer incidence in a population-based cohort of patients hospitalized with diabetes mellitus in Denmark. J Natl Cancer Inst 89:1360-1365, 1997

4. Hassan MM, Hwang LY, Hatten CJ, et al: Risk factors for hepatocellular carcinoma: Synergism of alcohol with viral hepatitis and diabetes mellitus. Hepatology 36:1206-1213, 2002

5. Klein $\mathrm{S}$, Wadden T, Sugerman HJ: AGA technical review on obesity. Gastroenterology 123:882932, 2002

6. Calle EE, Rodriguez C, Walker-Thurmond K, et al: Overweight, obesity, and mortality from cancer in a prospectively studied cohort of US adults. N Engl J Med 348:1625-1638, 2003

7. Samanic C, Chow WH, Gridley G, et al: Relation of body mass index to cancer risk in 362,552 Swedish men. Cancer Causes Control 17:901-909, 2006

8. Samanic C, Gridley G, Chow WH, et al: Obesity and cancer risk among white and black United States veterans. Cancer Causes Control 15:35-43, 2004

9. Moller $H$, Mellemgaard $A$, Lindvig $K$, et al: Obesity and cancer risk: A Danish record-linkage study. Eur J Cancer 30A:344-350, 1994

10. Pan SY, Johnson KC, Ugnat AM, et al: Association of obesity and cancer risk in Canada. Am J Epidemiol 159:259-268, 2004

11. Neuschwander-Tetri BA, Caldwell SH: Nonalcoholic steatohepatitis: Summary of an AASLD Single Topic Conference. Hepatology 37:1202-1219, 2003

12. Dixon JB, Bhathal PS, O'Brien PE: Nonalcoholic fatty liver disease: Predictors of nonalcoholic steatohepatitis and liver fibrosis in the severely obese. Gastroenterology 121:91-100, 2001

13. Andersen T, Gluud C: Liver morphology in morbid obesity: A literature study. Int J Obes 8:97106, 1984

14. García-Monzón C, Martín-Perez E, lacono OL, et al: Characterization of pathogenic and prognostic factors of nonalcoholic steatohepatitis associated with obesity. J Hepatol 33:716-724, 2000

15. Bruno $S$, Maisonneuve $P$, Castellana $P$, et al: Incidence and risk factors for non-alcoholic steatohepatitis: Prospective study of 5408 women enrolled in Italian tamoxifen chemoprevention trial. BMJ 330:932-935, 2005

16. Dam-Larsen S, Franzmann M, Andersen IB, et al: Long term prognosis of fatty liver: Risk of chronic liver disease and death. Gut 53:750-755, 2004

17. Adams LA, Lymp JF, St Sauver J, et al: The natural history of nonalcoholic fatty liver disease: A population-based cohort study. Gastroenterology 129:113-121, 2005

18. Yamada J, Tomiyama $H$, Yambe $M$, et al: Elevated serum levels of alanine aminotransferase and gamma glutamyltransferase are markers of inflammation and oxidative stress independent of the metabolic syndrome. Atherosclerosis 189:198-205, 2006

19. Yu MW, Gladek-Yarborough $A$, Chiamprasert S, et al: Cytochrome P450 2E1 and glutathione S-transferase M1 polymorphisms and susceptibility to hepatocellular carcinoma. Gastroenterology 109: 1266-1273, 1995

20. Yu MW, Chang HC, Liaw YF, et al: Familial risk of hepatocellular carcinoma among chronic hepatitis $\mathrm{B}$ carriers and their relatives. J Natl Cancer Inst 92:1159-1164, 2000

21. McLaughlin $T$, Reaven $G$, Abbasi $F$, et al: Is there a simple way to identify insulin-resistant individuals at increased risk of cardiovascular disease? Am J Cardiol 96:399-404, 2005

22. Rubin DB: Multiple imputation for nonresponse in surveys. New York, NY, John Wiley \& Sons, 1987

23. Becker U, Deis A, Sørensen TI, et al: Prediction of risk of liver disease by alcohol intake, sex, and age: A prospective population study. Hepatology 23:1025-1029, 1996

24. Morgan TR, Mandayam S, Jamal MM: Alcohol and hepatocellular carcinoma. Gastroenterology 127: S87-S96, 2004 (suppl)

25. Warram JH, Martin BC, Krolewski AS, et al: Slow glucose removal rate and hyperinsulinemia precede the development of type II diabetes in the offspring of diabetic parents. Ann Intern Med 113: 909-915, 1990

26. Chiasson $\mathrm{JL}$, Rabasa-Lhoret R: Prevention of type 2 diabetes: Insulin resistance and beta-cell function. Diabetes 53:S34-S38, 2004
27. Svegliati-Baroni G, Ridolfi F, Di Sario A, et al: Insulin and insulin-like growth factor-1 stimulate proliferation and type 1 collagen accumulation by human hepatic stellate cells: Differential effects on signal transduction pathways. Hepatology 29:17431751, 1999

28. Paradis $V$, Perlemuter $G$, Bonvoust $F$, et al: High glucose and hyperinsulinemia stimulate connective tissue growth factors expression: A potential mechanism involved in progression to fibrosis in nonalcoholic steatohepatitis. Hepatology 34:738744, 2001

29. Paradis V, Dargere D, Vidaud $M$, et al: Expression of connective tissue growth factor in experimental rat and human liver fibrosis. Hepatology 30:968-976, 1999

30. Ioannou GN, Weiss NS, Boyko EJ, et al: Contribution of metabolic factors to alanine aminotransferase activity in persons with other causes of liver disease. Gastroenterology 128:627-635, 2005

31. Bugianesi $E$, Marchesini $G$, Gentilcore $E$, et al: Fibrosis in genotype 3 chronic hepatitis $C$ and nonalcoholic fatty liver disease: Role of insulin resistance and hepatic steatosis. Hepatology 44:16481655, 2006

32. Stranges $S$, Dorn JM, Muti $P$, et al: Body fat distribution, relative weight, and liver enzyme levels: A population-based study. Hepatology 39:754-763, 2004

33. Cammà $\mathrm{C}$, Bruno $\mathrm{S}$, Di Marco V, et al: Insulin resistance is associated with steatosis in nondiabetic patients with genotype 1 chronic hepatitis $C$. Hepatology 43:64-71, 2006

34. Farrell GC, Larter CZ: Nonalcoholic fatty liver disease: From steatosis to cirrhosis. Hepatology 43:S99-S112, 2006

35. Yang S, Lin HZ, Hwang J, et al: Hepatic hyperplasia in noncirrhotic fatty livers: Is obesityrelated hepatic steatosis a premalignant condition? Cancer Res 61:5016-5023, 2001

36. Yang SQ, Lin HZ, Lane MD, et al: Obesity increases sensitivity to endotoxin liver injury: Implications for the pathogenesis of steatohepatitis. Proc Natl Acad Sci U S A 94:2557-2562, 1997

37. Kim KH, Shin HJ, Kim K, et al: Hepatitis B virus $X$ protein induces hepatic steatosis via transcriptional activation of SREBP1 and PPARgamma. Gastroenterology 132:1955-1967, 2007

38. Maheshwari A, Thuluvath PJ: Cryptogenic cirrhosis and NAFLD: Are they related? Am J Gastroenterol 101:664-668, 2006 